\title{
Brain-wide structural connectivity alterations under the control of Alzheimer risk genes
}

\section{Jingwen Yan* and Vinesh Raja V}

Department of BioHealth Informatics,

Indiana University Purdue University Indianapolis,

719 Indiana Ave, 46202, Indianapolis, USA

Email: jingyan@iupui.edu

Email: viv@iupui.edu

${ }^{*}$ Corresponding author

\section{Zhi Huang}

Electrical and Computing Engineering,

Indiana University Purdue University Indianapolis, 46202, Indianapolis, USA

Email: zhihuan@iu.edu

\section{Enrico Amico}

School of Industrial Engineering, Purdue Institute for Integrative Neuroscience,

Purdue University,

47907, West-Lafayette, USA

Email: eamico@purdue.edu

\section{Kwangsik Nho}

Radiology and Imaging Sciences,

Indiana University School of Medicine,

46202, Indianapolis, USA

Email: knho@iu.edu

\section{Shiaofen Fang}

\section{Computer Science,}

Indiana University Purdue University Indianapolis, 46202, Indianapolis, USA

Email: sfang@cs.iupui.edu 


\title{
Olaf Sporns
}

Psychological and Brain Sciences,

Indiana University,

47405, Bloomington, USA

Email: osporns@indiana.edu

\section{Yu-chien Wu and Andrew Saykin}

Radiology and Imaging Sciences,

Indiana University School of Medicine,

46202, Indianapolis, USA

Email: yucwu@iupui.edu

Email: asaykin@iupui.edu

\section{Joaquín Goñi}

School of Industrial Engineering,

Weldon School of Biomedical Engineering,

Purdue Institute for Integrative Neuroscience,

Purdue University,

47907, West-Lafayette, USA

Email: jgonicor@purdue.edu

\section{Li Shen}

Biostatistics, Epidemiology and Informatics, University of Pennsylvania,

19104, Philadelphia, USA

Email:Li.Shen@pennmedicine.upenn.edu

\begin{abstract}
Alzheimer's disease is the most common form of brain dementia characterised by gradual loss of memory. Large-scale genome-wide association studies (GWASs) have identified some AD risk genes, but their relationship with the brain-wide network breakdown in AD remains unknown. Using the genotype and diffusion tensor imaging (DTI) data in the Alzheimer's Disease Neuroimaging Initiative (ADNI) database, we performed a targeted genetic association analysis of three types of link measures, including fibre anisotropy, fibre length and density. For fair comparison, all link measures were normalised with zero mean and unit standard deviation. We focused on $34 \mathrm{AD}$ risk SNPs identified in previous GWAS studies. After Bonferroni correction, rs10498633 in SLC24A4 was found to be significantly associated with anisotropy, total number and length of fibres. rs429358 in top AD risk gene APOE showed nominal significance of association with the density of fibres between subcortical and cerebellum regions.
\end{abstract}

Keywords: brain connectivity; imaging genetics association; Alzheimer's disease. 
Reference to this paper should be made as follows: Yan, J., Vinesh Raja, V., Huang, Z., Amico, E., Nho, K., Fang, S., Sporns, O., Wu, Y-c., Saykin, A., Goñi, J. and Shen, L. (2020) 'Brain-wide structural connectivity alterations under the control of Alzheimer risk genes', Int. J. Computational Biology and Drug Design, Vol. 13, No. 1, pp.58-70.

Biographical notes: Jingwen Yan is an Assistant Professor of BioHealth Informatics at Indiana University Purdue University Indianapolis.

Vinesh Raja V is a Master student of BioHealth Informatics at Indiana University Purdue University Indianapolis.

Zhi Huang is a PhD student of Electrical and Computer Engineering at Indiana University Purdue University Indianapolis.

Enrico Amico is a postdoctoral fellow of Industrial Engineering at Purdue University.

Kwangsik Nho is an Assistant Professor of Radiology and Imaging Sciences at Indiana University School of Medicine.

Shiaofen Fang is a Professor of Computer Science at Indiana University Purdue University Indianapolis.

Olaf Sporns is a Professor of Psychological and Brain Sciences at Indiana University.

Yu-chien $\mathrm{Wu}$ is an Assistant Professor of Radiology and Imaging Sciences at Indiana University School of Medicine.

Andrew Saykin is a Professor of Radiology and Imaging Sciences at Indiana University School of Medicine.

Joaquín Goñi is an Assistant Professor of Industrial Engineering at Purdue University.

Li Shen is a Professor of Informatics in Department of Biostatistics, Epidemiology and Informatics at University of Pennsylvania.

This paper is a revised and expanded version of a paper entitled 'Brain-wide structural connectivity alterations under the control of Alzheimer risk genes' presented at International Conference on Intelligent Biology and Medicine, Los Angeles, 10-12 June, 2018.

\section{Background}

Alzheimer's disease is the most common form of brain dementia characterised by gradual loss of memory followed by further deterioration of other cognitive function. It has become one of the leading cause of death and is still increasingly affecting the ageing population nationwide (Association et al., 2017). Due to the lack of effective treatment, number of 
deaths due to Alzheimer's between 2000 and 2014 has significantly increased (Association et al., 2017).

Neuroimaging has been a major approach to study AD which allows mapping of structural, functional and molecular AD pathology inside brain (Teipel et al., 2015). Accumulating evidence from previous studies suggests abnormal imaging patterns in AD patients, such as cortical and subcortical atrophy (Fox and Schott, 2004), cortical amyloid deposition (Villemagne et al., 2011), grey matter atrophy (Thompson et al., 2003), and functional cortical disconnection (Pievani et al., 2011). Recently, there is a growing interest in structural brain connectivity captured through diffusion tensor imaging (DTI) in AD. AD patients and those in a mild stage were found to have loss of inter-hemisphere connectivities (Wang et al., 2015) and increased diffusion anisotropy (Douaud et al., 2011).

On the other hand, genetic factors play an essential role in AD. Results from large-scale twin studies suggest the heritability of AD to reach 70-80\% (Wingo et al., 2012; Sleegers et al., 2010). Originally identified in several targeted studies (Corder et al., 1993, 1994; Talbot et al., 1994; Saunders et al., 1993), the e 4 allele of $A P O E$ is the most well-known genetic variation associated with increased $\mathrm{AD}$ risk and has been confirmed repeatedly in multiple populations around the world. Later, large-scale genome-wide association studies (GWASs) identified and validated 20 novel risk genetic loci (Lambert et al., 2013; Hollingworth et al., 2011; Harold et al., 2009; Naj et al., 2011; Seshadri et al., 2010), which have been recently found to differentially regulate brain amyloidosis across different disease stages (Apostolova et al., 2018). However, how they exert effect on the brain-wide breakdown of structural brain connectivity has not been studied yet.

Leveraging the genotype and DTI data in the Alzheimer's disease neuroimaging initiative (ADNI) (Weiner et al., 2010; Saykin et al., 2015), in this paper, we perform a targeted genetic association analysis of brain-wide connectivity measures to discover the brain network alterations under the control of AD. We focus our analysis on link level measures, including fibre anisotropy, fibre length and density. In addition, to avoid potential bias introduced in imaging processing pipeline, we repeat our imaging processing pipeline and evaluate the reliability of all connectivity measures. Only highly reliable connectivity measures will be considered for the further imaging genetics association analysis. Using age at scan and gender as covariates, we employ general linear regression models to investigate the association between each pair of candidate SNP and connectivity measure. After enforcing the stringent Bonferroni correction, rs10498633 in SLC24A4 was found to be significantly associated with anisotropy, total number and length of fibres including some connecting hemispheres, which is consistent with existing findings. rs429358 in top AD risk gene $A P O E$ only shows nominal significance of association with the density of fibres between Subcortical and Cerebellum $\left(p=2.71 \times 10^{-6}\right)$.

\section{Methods}

\subsection{ADNI Cohort}

Data used in this study were obtained from the Alzheimer's disease neuroimaging initiative (ADNI) database (Saykin et al., 2015; Weiner et al., 2010). Involving researchers from more than 50 sites in the USA and Canada, the ADNI aims to track the progression of AD in the human brain by collecting longitudinal neuroimaging, biochemical, and genetic biological data. A key aim of the ADNI is to provide the opportunity to combine genetics with 
multiple types of imaging (e.g., magnetic resonance imaging (MRI) and positron emission tomography (PET)) and clinical data to help investigate mechanisms of the disease. For up-to-date information, see www.adni-info.org. For the proposed brain-wide connectomics genetics association analysis, we downloaded the structural MRI (sMRI) scans, DTI scans, GWAS genotype and demographic data from the ADNI website. Written informed consent was obtained at the time of enrolment and/or genetic sample collection and protocols were approved by each participating study and sites' Institutional Review Board.

\subsection{Brain connectivity measures}

The DTI data was first denoised and corrected for motion and distortion using the approach described in a previous study (Manjón et al., 2013). Tractography was performed in Camino (Cook et al., 2006) based on white matter fibre orientation distribution function (ODF). Streamlines were modelled with a multi-tensor modelling approach, where voxels will fit up to two fibre orientations. Second, the sMRI scans were registered to the b0 volume of DTI data using the FNIRT toolbox (Jenkinson et al., 2012) and 278 brain regions of interest (ROIs) were extracted following (Shen et al., 2013). The final networks between 278 brain ROIs were constructed using fibres going through white matter and connecting ROIs. In this project, we focus on the link level measures and calculated the fibre anisotropy (FA), length of fibres (LOF) and number of the fibres (NOF) connecting each pair of ROIs. Considering that the number of fibres are partially dependent on the surface area of their connected ROIs, we derived a new measure, the fibre density (FD), for the following association analysis, which is the fraction between number of fibres and the average surface of grey-matter regions $i$ and $j$.

\subsection{Reliability test}

Considering that some steps in the DTI processing pipeline are subject to random factors, e.g., when identifying the streamlines, the initial seed selection may lead to different results, we further evaluated the reliability of all brain connectivity measures. Since each subject only have one DTI scan in ADNI, we repeated our imaging processing pipeline three times, without changing any parameters, and quantified the reliability of FA, FD and LOF measures by calculating their intraclass correlation coefficients (ICC) across three runs.

\subsection{Genotype data}

Genotyping was performed using the Illumina HumanOmni Express BeadChip for all participants included. We first performed standard sample and SNP quality control procedures as described previously (Nho et al., 2013). The un-genotyped SNPs were imputed using $\mathrm{MACH}$ and minimac in a two-stage procedure following a previous study (Nho et al., 2015). The pilot 1 data of the 1000 Genomes Project were used as a reference panels for inferring missing genotypes. Minimac produced the posterior probabilities of the imputed genotypes at un-genotyped marker loci for each individual. To assure the quality of imputation, an $r^{2}$ value equal to 0.30 was imposed as the threshold to filter the imputed genotypes. In this project, since we are particularly interested in the brain network alterations under the control of AD, rs429358 in APOE and 33 AD risk SNPs used in Apostolova et al. (2018) were included for the association analysis (Table 1). 
Table 134 AD risk loci included in the brain-wide connectomics genetics association

\begin{tabular}{lccccc}
\hline SNP & Gene & MAF & SNP & Gene & MAF \\
\hline rs3752246 & ABCA7 & 0.17 & rs6701713 & CRI & 0.25 \\
rs3764650 & ABCA7 & 0.2 & rs11767557 & EPHA1 & 0.2 \\
rs4147929 & ABCA7 & 0.18 & rs11771145 & EPHA1 & 0.43 \\
rs6733839 & BIN1 & 0.39 & rs17125944 & FERMT2 & 0.11 \\
rs744373 & BIN1 & 0.36 & rs35349669 & INPP5D & 0.21 \\
rs7561528 & BIN1 & 0.2 & rs190982 & MEF2C & 0.22 \\
rs7274581 & $C A S S 4$ & 0.09 & rs610932 & MS4A6A & 0.45 \\
rs9349407 & $C D 2 A P$ & 0.19 & rs983392 & MS4A6A & 0.23 \\
rs10948363 & $C D 2 A P$ & 0.19 & rs2718058 & NME8 & 0.34 \\
rs3865444 & $C D 33$ & 0.21 & rs3851179 & PICALM & 0.31 \\
rs10838725 & $C E L F 1$ & 0.26 & rs10792832 & PICALM & 0.31 \\
rs11136000 & $C L U$ & 0.38 & rs561655 & PICALM & 0.34 \\
rs1532278 & $C L U$ & 0.26 & rs28834970 & PTK2B & 0.32 \\
rs9331896 & $C L U$ & 0.38 & rs10498633 & SLC24A4/RIN3 & 0.15 \\
rs12034383 & $C R 1$ & 0.41 & rs1131497 & SORL1 & 0.37 \\
rs3818361 & $C R 1$ & 0.25 & rs1476679 & ZCWPW1 & 0.21 \\
rs6656401 & $C R 1$ & 0.07 & rs429358 & APOE & 0.15 \\
\hline
\end{tabular}

\subsection{Brain connectomics genetics association}

We performed a targeted genetic association analysis between each pair of SNP and link level feature by employing a general linear model (GLM) approach in R. Shown in equation (1) is the final linear model we applied with age at scan and gender as independent variables. Here, $i \in\{1,2, \ldots, 82321\}$ and $j \in\{1,2, \ldots, 34\}$. Lin $k_{i}$ is the $i$ th link level measure, e.g., fibre anisotropy of the link between two brain ROIs in Shen atlas. For fair comparison of genetic effect on different measures, FA, LOF and FD measures were normalised to have zero mean and standard deviation as one. In the subsequent analysis of brain connectivity to examine the association between candidate SNPs and brain-wide connectivity measures, multiple comparison correction was enforced using the Bonferroni method at a 0.05 level of significance with the total number of test estimated to be $82,321 \times 34=2,798,914$.

$$
\operatorname{Link}_{i}=\text { Age }+ \text { Gender }+S N P_{j}
$$

\section{Results}

\subsection{Subject}

All the subjects included in this study are participants from the ADNI-2 and ADNI-GO stages. Among all 273 Caucasian subjects with DTI scans, 178 of them without missing values in sMRI scans, genotype of 34 risk SNPs and demographic information were kept for the association analysis. In total, the study population is consisted of 34 healthy controls (HC), 26 individuals with subjective memory complain (SMC), 59 individuals with early mild cognitive impairment (EMCI), 23 individuals with late MCI (LMCI) and 36 individuals with AD. Shown in Table 2 is the detailed demographic information for all 178 subjects. 
Table 2 Demographic information of all participants

\begin{tabular}{lccccc}
\hline & $H C$ & $S M C$ & $E M C I$ & $L M C I$ & $A D$ \\
\hline Number & 34 & 26 & 59 & 23 & 36 \\
Gender(M/F) & $19 / 15$ & $18 / 8$ & $34 / 25$ & $16 / 7$ & $22 / 14$ \\
Age(Mean \pm std) & $72.97 \pm 5.94$ & $73.5 \pm 5.22$ & $72.9 \pm 7.59$ & $71.39 \pm 8.1$ & $75.06 \pm 8.94$ \\
\hline
\end{tabular}

\subsection{Reliability test}

Among all brain connectivity measures, including FA, LOF and FD, about one third of the measures show inconsistency across three runs with ICC smaller than 0.9. More specifically, 26,886 out of 38,503 FA measures $(69.83 \%)$ passed the reliability test with ICC greater than 0.9 (Koo and Li, 2016). For LOF and FD measures, there are 25,644 (66.6\%) and $29,791(77.37 \%)$ passing the same threshold. Shown in Figure 1 is the ICC distribution for FA, LOF and FD respectively. This similar reliability distribution is within our expectation since the major random effect that leads to the reliability issue is the seed selection when identifying streamlines, which however does not exert too much effect on the subsequent link level measure extraction. In this paper, we only included those measures $(N=26,886+$ $25,644+29,791=82,321)$ with excellent reliability (ICC $\geq 0.9)$ for further analysis.

Figure 1 ICC distribution for all measures: (a) fibre anisotropy; (b) length of fibres and (c) fibre density (see online version for colours)
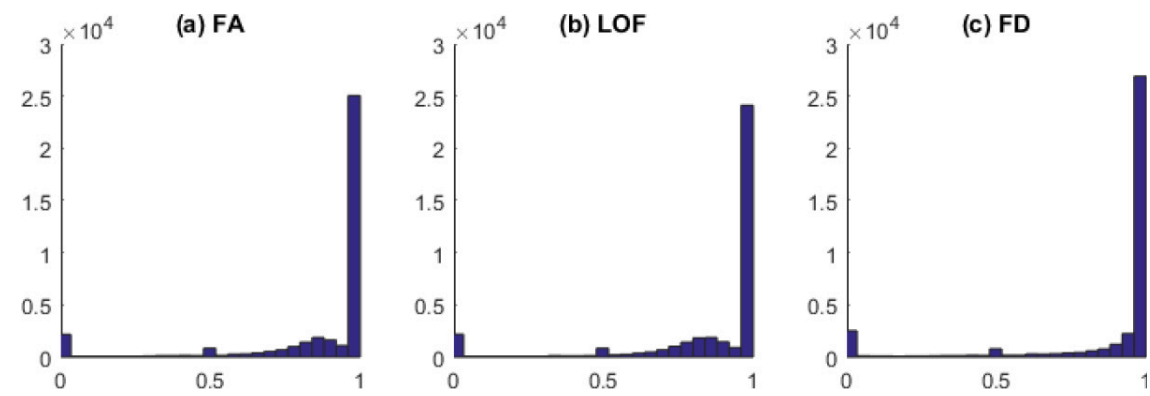

\subsection{Genetic effect of $A D$ risk genes on connectivity measures}

We identified several significant associations between 34 AD risk loci and three types of connectivity measures with excellent reliability (Table 3). For better interpretation of results, we mapped 278 brain ROIs to Yeo parcellation with seven groups (3rd and 4th columns in Table 3), including visual (VIS), Somato-Motor (SM), Dorsal Attention (DA), Ventral Attention(VA), Limbic system (L), Fronto-Parietal (FP), and Default Mode Network (DMN). These seven brain groups/networks are identified as functionally distinct cortical regions in on a clustering analysis of resting-state functional connectivity MRI (Yeo et al., 2011). Shown in Figure 2(a) is the brain map of Yeo atlas we used in MNI 152 space. We also added subcortical regions (SUBC) and cerebellum (CER) to complement Yeo atlas using the strategy previously described in Amico et al. (2017). Each Yeo ROI consists of multiple Shen ROIs. 
Figure 2 Heatmap of all SNP-connectivity associations and brain map of selected connectivities with uncorrected $\mathrm{p} \leq 5 \mathrm{e}-6$ : (a) Brain map of Yeo parcellation with 7 groups in MNI 152 space; (b) Top panel: Heatmap showing the association of rs10498633 in SLC24A4 with three different types of connectivity measures. Rows and columns are reordered to form 7 groups corresponding to Yeo parcellation. Top and side colourbar indicate the corresponding Yeo parcellation of each ROI. The last two groups, subcortical (SUBC) and Cerebellum (CER), are added to complement the Yeo atlas; (c) Brain map of the association between rs17125944 and FA measures; (d) Brain map of the association between rs7274581 and LOF measures and (e) Brain map of the association between rs429358 and FD measures. All the links in the brain connectivity map share the same colourmap with the dots in the heatmap. Yeo parcellation in (a), top and side colourbars in (b), and all the nodes in the brain connectivity map share the same colour scheme
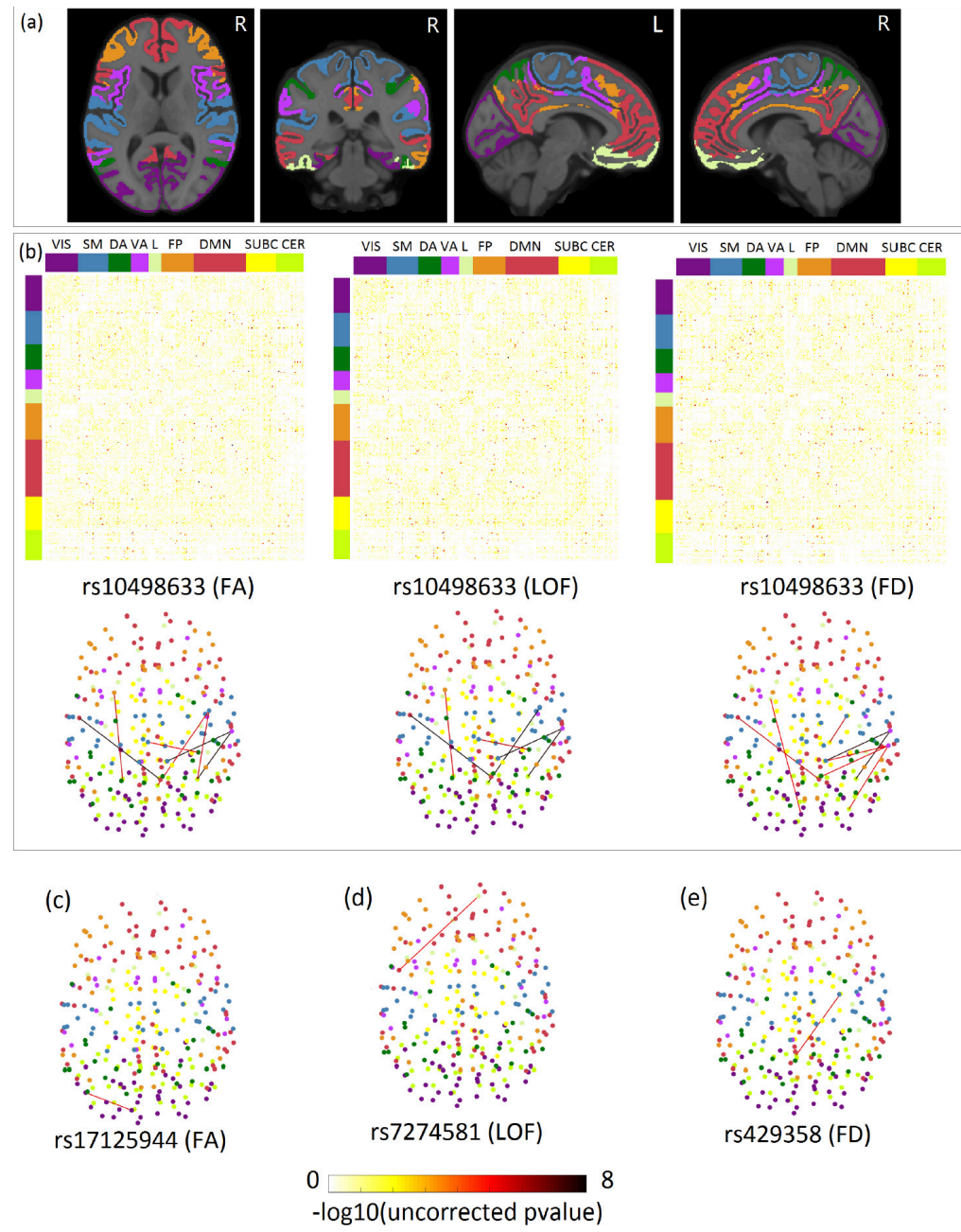

rs10498633 (FA)

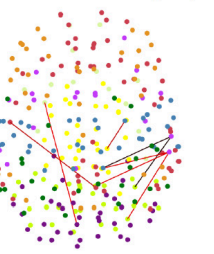

-log10(uncorrected pvalue) (e)

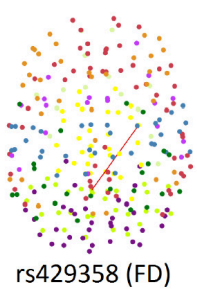

For FA measure, the genetic effect of rs10498633 in SLC24A4 achieved brain-wide significance after enforcing the stringent Bonferroni correction based on the total number of connectomic measures and risk SNPs $(p=0.05 /(26886+25644+29791) \times 34=2 \times$ $10^{-8}$ ). It showed association with the anisotropy of fibres connecting Ventral Attention 
and Subcortical, Ventral Attention and Cerebellum, and within Default Mode Network respectively. rs 10498633 in SLC24A4 is also found to be significantly associated with the length and density of fibres connecting Cerebellum and Somato-Motor, Ventral Attention and Cerebellum, Ventral Attention and Subcortical, and within Default Mode Network etc. In addition, other SNPs such as rs11771145 and rs10498633 are found to be nominally associated with the fibres connecting Cerebellum and Somato-Motor, Dorsal Attention and Somato-Motor respectively. rs 429358 in top AD risk gene APOE only shows nominal significance of association with the density of fibres between Subcortical and Cerebellum $\left(p=2.71 \times 10^{-6}\right)$.

Shown in the top panel of Figure 2(b) is the heatmap of association results between rs 10498633 and three types of connectivity measures. Each row and column were reordered based on Yeo atlas. The bottom panel is the corresponding brain connectivity map showing only links with ICC $\geq 0.9$ and uncorrected $\mathrm{p} \leq 5 \mathrm{e}-6$. Links in the bottom panel are corresponding to the dots in the top panel. Different node colours in the brain connectivity map indicate the Yeo group information of each ROI. Among all the fibres affected by those risk genes, we observed that some of them associated with rs 10498633 connect two hemispheres. This is consistent with previous findings that $\mathrm{AD}$ patients show loss of interhemispheric connectivity. Figure 2(c)-(e) are example brain maps of three associations with nominal significance.

\section{Discussion}

To the best of our knowledge, this is the first comprehensive analysis to test the association of the top $\mathrm{AD}$ risk variants with brain connectivity measures. By performing a targeted genetic association of brain-wide connectivity, we were able to replicate the previous findings such as abnormal inter-hemispheric connectivity patterns and fibre anisotropy in AD. rs 10498633 in $S L C 24 A 4$ consistently shows significant genetic effect on all three type of brain connectivity measures, including some inter-hemispheric connectivity which is a known abnormal pattern in $\mathrm{AD}$ patients.

$S L C 24 A 4$ encodes a member of the potassium-dependent sodium/calcium exchanger protein family. It has been previously reported to have significant association with grey matter density, brain metabolism (Stage et al., 2016) and DNA methylation in prefrontal cortex (Yu et al., 2015). However, due to the limited access to brain tissues, there is few studies looking into the role of $S L C 24 A 4$ in brain and our knowledge in that part is still very limited. Recently emerging databases in tissue-specific gene expression and expression quantitative trait (eQTL), however, allow us to peek into this from a different perspective. According to the Allen Human Brain Atlas (AHBA) (www.brain-map.org), SLC24A4 is highly expressed in part of the cerebellum and some subcortical regions, including Amygdala, Putamen, etc. This was later confirmed in several other emerging databases, such as the genotype-tissue expression project (GTEx) (www.gtexportal.org), the Human Protein Atlas (HPA) (www.proteinatlas.org), and the Functional Annotation of The Mammalian Genome (FANTOM) (fantom.gsc.riken.jp). In particular, it was consistently found that the RNA expression of $S L C 24 A 4$ is very selectively high in hippocampus, a subcortical region that plays a key role in memory performance and is well known to be AD-relevant. All of these enriched brain regions were consistent with our findings that the links connecting these regions are affected by the SNP rs 10498633 in SLC24A4. In addition, another large-scale eQTL analysis (www.braineac.org) show that rs 10498633 is an eQTL in several subcortical 
regions and white matter where the fibres are located. Taken together, these evidence gives strong support to our connectomics-genetics association findings. It also suggests potential role of rs10498633 in regulating the brain connectivity by mediating the expression of certain genes including SLC24A4.

Table 3 Top five associations between AD risk SNPs and brain connectivity measures.

\begin{tabular}{lcccccr}
\hline Data type & SNP & Yeo ROI1 & Yeo ROI2 & Beta & $p$ (uncorrected) & ICC \\
\hline Fibre & rs10498633 & DMN & DMN & 0.89 & $1.44 \mathrm{E}-08$ & 1.00 \\
anisotropy & rs10498633 & VA & CER & 0.88 & $1.68 \mathrm{E}-08$ & 1.00 \\
& rs10498633 & VA & SUBC & 0.88 & $1.73 \mathrm{E}-08$ & 1.00 \\
& rs10498633 & CER & FP & 0.76 & $1.52 \mathrm{E}-06$ & 1.00 \\
& rs10498633 & CER & SM & 0.75 & $2.08 \mathrm{E}-06$ & 1.00 \\
\hline Length & rs10498633 & CER & SM & 0.90 & $8.73 \mathrm{E}-09$ & 1.00 \\
of fibres & rs10498633 & DMN & DMN & 0.90 & $9.06 \mathrm{E}-09$ & 1.00 \\
& rs10498633 & VA & CER & 0.88 & $1.68 \mathrm{E}-08$ & 1.00 \\
& rs10498633 & VA & SUBC & 0.88 & $1.68 \mathrm{E}-08$ & 1.00 \\
& rs10498633 & DA & SM & 0.76 & $1.54 \mathrm{E}-06$ & 1.00 \\
\hline Fibre & rs10498633 & VA & CER & 0.88 & $1.76 \mathrm{E}-08$ & 1.00 \\
density & rs10498633 & VA & SUBC & 0.88 & $2.20 \mathrm{E}-08$ & 1.00 \\
& rs4147929 & SUBC & CER & 0.68 & $1.04 \mathrm{E}-06$ & 1.00 \\
& rs10498633 & SM & SUBC & 0.77 & $1.40 \mathrm{E}-06$ & 0.95 \\
& rs10498633 & DMN & DMN & 0.76 & $1.48 \mathrm{E}-06$ & 1.00 \\
\hline
\end{tabular}

As an exploratory and targeted imaging genetics analysis, this study has several limitations. First, since we only focused on three link-level measures and one third of the links are excluded due to poor reliability, there may be many other significant associations beyond what we have reported. Also, the connectomic features are treated individually without considering their correlation structure. Therefore the Bonferroni significance level may be overly conservative. Second, due to the small sample size, this study explored the imaging genetics association by coupling all diagnosis groups together and cannot reveal the group specific associations between connectomic and genetic variations, which is another important research topic for future investigation. Finally, due to the lack of data, we were not able to perform the validation analysis. However, recent release of genotype data from the Human Connectome Project makes this possible and we will validate our findings using this cohort in the near future.

\section{Conclusion}

We performed a targeted genetic association analysis of brain-wide connectivity measures to investigate the effect of AD risk genes on brain networks. We identified several significant genetics-connectomics associations. Particularly, rs 10498633 in SLC24A4 shows significant genetic effect on the anisotropy, length and density of fibres, which connect subcortical regions, cerebellum and part of the cerebral cortex regions involved in the default mode network. Given that SLC24A4 is highly expressed in those regions and rs10498633 is an eQTL locus of SLC24A4 in subcortical brain regions, we hypothesise that the regulation role of rs 10498633 in brain network may be achieved by mediating the expression of certain genes including SLC24A4. 


\section{Acknowledgements}

This research was supported by NIH grants R01 LM012535, R03 AG054936, R01 AG053993, R01 EB022574, R01 LM011360, R01 MH108467, R01 AG019771, P30 AG010133 and K01 AG049050. This project was also funded, in part, with support from the Indiana Clinical and Translational Sciences Institute funded, in part by Grant Number UL1TR001108 from the National Institutes of Health, National Center for Advancing Translational Sciences, Clinical and Translational Sciences Award.

\section{References}

Amico, E., Marinazzo, D., Di Perri, C., Heine, L., Annen, J., Martial, C., Dzemidzic, M., Kirsch, M., Bonhomme, V., Laureys, S. and Goñi, J. (2017) 'Mapping the functional connectome traits of levels of consciousness', NeuroImage, Vol. 148, pp.201-211.

Apostolova, L.G., Risacher, S.L., Duran, T., Stage, E.C., Goukasian, N., West, J.D., Do, T.M., Grotts, J., Wilhalme, H., Nho, K. and Phillips, M. (2018) 'Associations of the Top 20 Alzheimer disease risk variants with brain amyloidosis', JAMA Neurology, Vol. 75, No. 3, pp.328-341.

Alzheimer's Association (2017) '2017 Alzheimer's disease facts and figures', Alzheimer's and Dementia, Vol. 13, No. 4, pp.325-373.

Cook, P., Bai, Y., Nedjati-Gilani, S., Seunarine, K., Hall, M., Parker, G. and Alexander, D. (2006) 'Camino: open-source diffusion-mri reconstruction and processing', 14th Scientific Meeting of the International Society for Magnetic Resonance in Medicine, Vol. 2759, Seattle WA, USA.

Corder, E.H., Saunders, A.M., Strittmatter, W.J., Schmechel, D.E., Gaskell, P.C., Small, G., Roses, A.D., Haines, J. and Pericak-Vance, M.A. (1993) 'Gene dose of apolipoprotein e type 4 allele and the risk of alzheimer's disease in late onset families', Science, Vol. 261, No. 5123, pp.921-923.

Corder, E.H., Saunders, A.M., Risch, N.J., Strittmatter, W.J., Schmechel, D.E., Gaskell, P.C., Rimmler, J.B., Locke, P.A., Conneally, P.M., Schmader, K.E. and Small, G.W. (1994) 'Protective effect of apolipoprotein E type 2 allele for late onset Alzheimer disease', Nature Genetics, Vol. 7, No. 2, pp.180-184.

Douaud, G., Jbabdi, S., Behrens, T.E., Menke, R.A., Gass, A., Monsch, A.U., Rao, A., Whitcher, B., Kindlmann, G., Matthews, P.M. and Smith, S. (2011) 'DTI measures in crossing-fibre areas: increased diffusion anisotropy reveals early white matter alteration in MCI and mild Alzheimer's disease', Neuroimage, Vol. 55, No. 3, pp.880-890.

Fox, N.C. and Schott, J.M. (2004) 'Imaging cerebral atrophy: normal ageing to alzheimer's disease', The Lancet, Vol. 363, No. 9406, pp.392-394.

Harold, D., Abraham, R., Hollingworth, P., Sims, R., Gerrish, A., Hamshere, M.L., Pahwa, J.S., Moskvina, V., Dowzell, K., Williams, A. and Jones, N. (2009) 'Genome-wide association study identifies variants at CLU and PICALM associated with Alzheimer's disease', Nature Genetics, Vol. 41, No. 10, p.1088.

Hollingworth, P., Harold, D., Sims, R., Gerrish, A., Lambert, J.C., Carrasquillo, M.M., Abraham, R., Hamshere, M.L., Pahwa, J.S., Moskvina, V. and Dowzell, K. (2011) 'Common variants at ABCA7, MS4A6A/MS4A4E, EPHA1, CD33 and CD2AP are associated with Alzheimer's disease', Nature Genetics, Vol. 43, No. 5, p.429.

Jenkinson, M., Beckmann, C.F., Behrens, T.E., Woolrich, M.W. and Smith, S.M. (2012) 'Fsl', Neuroimage, Vol. 62, No. 2, pp.782-790.

Koo, T.K. and Li, M.Y. (2016) 'A guideline of selecting and reporting intraclass correlation coefficients for reliability research', Journal of Chiropractic Medicine, Vol. 15, No. 2, pp.155-163. 
Lambert, J-C., Ibrahim-Verbaas, C.A., Harold, D., Naj, A.C., Sims, R., Bellenguez, C., Jun, G., DeStefano, A.L., Bis, J.C., Beecham, G.W. et al. (2013) 'Meta-analysis of 74,046 individuals identifies 11 new susceptibility loci for alzheimer's disease', Nature genetics, Vol. 45, No. 12, p.1452.

Manjón, J.V., Coupé, P., Concha, L., Buades, A., Collins, D.L. and Robles, M. (2013) 'Diffusion weighted image denoising using overcomplete local pca', PloS one, Vol. 8, No. 9, pp.e73021.

Naj, A.C., Jun, G., Beecham, G.W., Wang, L.S., Vardarajan, B.N., Buros, J., Gallins, P.J., Buxbaum, J.D., Jarvik, G.P., Crane, P.K. and Larson, E.B. (2011) 'Common variants at MS4A4/MS4A6E, CD2AP, CD33 and EPHA1 are associated with late-onset Alzheimer's disease', Nature Genetics, Vol. 43, No. 5, p.436.

Nho, K., Corneveaux, J.J., Kim, S., Lin, H., Risacher, S.L., Shen, L., Swaminathan, S., Ramanan, V.K., Liu, Y., Foroud, T. and Inlow, M.H. (2013) 'Whole-exome sequencing and imaging genetics identify functional variants for rate of change in hippocampal volume in mild cognitive impairment', Molecular Psychiatry, Vol. 18, No. 7, p.781.

Nho, K., Kim, S., Risacher, S.L., Shen, L., Corneveaux, J.J., Swaminathan, S., Lin, H., Ramanan, V.K., Liu, Y., Foroud, T.M. and Inlow, M.H. (2015) 'Protective variant for hippocampal atrophy identified by whole exome sequencing', Annals of Neurology, Vol. 77, No. 3, pp.547-552.

Pievani, M., de Haan, W., Wu, T., Seeley, W.W. and Frisoni, G.B. (2011) 'Functional network disruption in the degenerative dementias', The Lancet Neurology, Vol. 10, No. 9, pp.829-843.

Saunders, A.M., Strittmatter, W.J., Schmechel, D., George-Hyslop, P.S., Pericak-Vance, M.A., Joo, S.H., Rosi, B.L., Gusella, J.F., Crapper-MacLachlan, D.R., Alberts, M.J. and Hulette, C. (1993) 'Association of apolipoprotein E allele ?4 with late?onset familial and sporadic Alzheimer's disease', Neurology, Vol. 43, No. 8, pp.1467-1467.

Saykin, A.J., Shen, L., Yao, X., Kim, S., Nho, K., Risacher, S.L., Ramanan, V.K., Foroud, T.M., Faber, K.M., Sarwar, N. and Munsie, L.M. (2015) 'Genetic studies of quantitative MCI and AD phenotypes in ADNI: Progress, opportunities, and plans', Alzheimer's and Dementia, Vol. 11, No. 7, pp.792-814.

Seshadri, S., Fitzpatrick, A.L., Ikram, M.A., DeStefano, A.L., Gudnason, V., Boada, M., Bis, J.C., Smith, A.V., Carrasquillo, M.M., Lambert, J.C. and Harold, D. (2010) 'Genome-wide analysis of genetic loci associated with Alzheimer disease', Jama, Vol. 303, No. 18, pp.1832-1840.

Shen, X., Tokoglu, F., Papademetris, X. and Constable, R.T. (2013) 'Groupwise whole-brain parcellation from resting-state fmri data for network node identification', Neuroimage, Vol. 82, pp.403-415.

Sleegers, K., Lambert, J-C., Bertram, L., Cruts, M., Amouyel, P. and Van Broeckhoven, C. (2010) 'The pursuit of susceptibility genes for alzheimer's disease: progress and prospects', Trends in Genetics, Vol. 26, No. 2, pp.84-93.

Stage, E., Duran, T., Risacher, S.L., Goukasian, N., Do, T.M., West, J.D., Wilhalme, H., Nho, K., Phillips, M., Elashoff, D. and Saykin, A.J. (2016) 'The effect of the top 20 Alzheimer disease risk genes on gray-matter density and FDG PET brain metabolism', Alzheimer's and Dementia: Diagnosis, Assessment and Disease Monitoring, Vol. 5, pp.53-66.

Talbot, C., Lendon, C., Craddock, N., Shears, S., Morris, J. and Goate, A. (1994) 'Protection against alzheimer's disease with apoe e2', The Lancet, Vol. 343, No. 8910, pp.1432-1433.

Teipel, S., Drzezga, A., Grothe, M.J., Barthel, H., ChÃ@telat, G., Schuff, N., Skudlarski, P., Cavedo, E., Frisoni, G.B., Hoffmann, W. and Thyrian, J.R. (2015) 'Multimodal imaging in Alzheimer's disease: validity and usefulness for early detection', The Lancet Neurology, Vol. 14, No. 10, pp.1037-1053.

Thompson, P.M., Hayashi, K.M., De Zubicaray, G., Janke, A.L., Rose, S.E., Semple, J., Herman, D., Hong, M.S., Dittmer, S.S., Doddrell, D.M. et al. (2003) 'Dynamics of gray matter loss in alzheimer's disease', Journal of Neuroscience, Vol. 23, No. 3, pp.994-1005. 
Villemagne, V.L., Ong, K., Mulligan, R.S., Holl, G., Pejoska, S., Jones, G., O’Keefe, G., Ackerman, U., Tochon-Danguy, H., Chan, J.G. and Reininger, C.B. (2011) 'Amyloid imaging with 18F-florbetaben in Alzheimer disease and other dementias', Journal of Nuclear Medicine, pp.jnumed-111.

Wang, Z., Wang, J., Zhang, H., Mchugh, R., Sun, X., Li, K. and Yang, Q.X. (2015) 'Interhemispheric functional and structural disconnection in alzheimer's disease: a combined resting-state fmri and dti study', PLoS One, Vol. 10, No. 5, pp.e0126310.

Weiner, M.W., Aisen, P.S. et al. (2010) 'The alzheimer's disease neuroimaging initiative: progress report and future plans', Alzheimers Dement, Vol. 6, No. 3, pp.202-211 e7.

Wingo, T.S., Lah, J.J., Levey, A.I. and Cutler, D.J. (2012) 'Autosomal recessive causes likely in early-onset alzheimer disease', Archives of neurology, Vol. 69, No. 1, pp.59-64.

Yeo, B.T., Krienen, F.M., Sepulcre, J., Sabuncu, M.R., Lashkari, D., Hollinshead, M., Roffman, J.L., Smoller, J.W., Zollei, L., Polimeni J.R., Fischl, B., Liu, H. and Buckner, R.L. (2011) 'The organization of the human cerebral cortex estimated by intrinsic functional connectivity', J Neurophysiol., Vol. 106, No. 3, pp.1125-1165.

Yu, L., Chibnik, L.B., Srivastava, G.P., Pochet, N., Yang, J., Xu, J., Kozubek, J., Obholzer, N., Leurgans, S.E., Schneider, J.A. and Meissner, A. (2015) 'Association of brain DNA methylation in SORL1, ABCA7, HLA-DRB5, SLC24A4, and BIN1 with pathological diagnosis of Alzheimer disease', JAMA Neurology, Vol. 72, No. 1, pp.15-24. 